\title{
GESTIÓN DE MEDIOS COMUNITARIOS Y POPULARES: CASO RADIO COMUNITARIA DE LA NACIONALIDAD AWÁ DEL ECUADOR
}

\author{
Management of community and popular media: the case of the community radio of \\ the "Awá" nationality in Ecuador.
}

Maria B. Ávalos-Torres ${ }^{1}$

Ana M. Culqui-Medina ${ }^{2}$

\section{Resumen}

En el presente trabajo se analiza el modelo de gestión integral comunicacional desarrollado para la radio comunitaria de la nacionalidad Awá del Ecuador, frecuencia otorgada por el gobierno en 2009. Se plantean categorías teóricas de la Escuela Latinoamericana de Comunicación, que cuestionan esquemas verticalestradicionalistas, y se toma como base el modelo de Comunicación Horizontal de Luis Ramiro Beltrán, donde el diálogo es catalizador de procesos democráticos y participativos: la radio se convierte en el promotor directo de diálogo. Se utilizó una combinación de metodología cuantitativa- cualitativa. Se realizaron encuestas a una muestra de la población Awá en tres provincias para determinar su contexto, costumbres, cultura, cosmovisión; además, entrevistas a profundidad a los dirigentes de la radio y miembros de la Federación de Centros Awá del Ecuador. Los resultados corroboraron que un medio comunitario debe seguir un proceso de estructuración, y el "Modelo de Gestión" resultado de esta investigación permitió a la radio una mejor organización, sostenibilidad y licencia definitiva.

\section{Palabras clave}

Democratización de la palabra; participación; visibilización, nacionalidad Awá, comunicación horizontal..

\section{Abstract}

In this research, the management model developed for the community radio of the indigenous nationality "Awá" of Ecuador is analyzed, the Radio frequency was awarded by the government in 2009. Theoretical categories of the Latin American School of Communication are proposed, which question vertical-traditionalist schemes; the model of Horizontal Communication of Luis Ramiro Beltrán is taken as a basis, where the dialogue is a catalyst for democratic and participatory processes: the radio becomes the direct promoter of the dialogue. A combination of quantitative-qualitative methodology was used. Surveys on a sample population of "Awá" community in three provinces of Ecuador, to determine its context, customs, culture, worldview and depth interviews to the leaders of the radio and members of Federation of "Awá" Centers of Ecuador were conducted. The results corroborated that a community radio must follow a structuring process, and the "Management Model"; also, this result allowed the radio to have a better organization, sustainability and definite radio station license.

\section{Key words}

Community media; democratization of the word; participation; visibility, Awá nationality.

\footnotetext{
${ }^{1}$ Universidad Nacional de Chimborazo (Ecuador). Correo electrónico: maria.avalos@unach.edu.ec.

${ }^{2}$ Pontificia Universidad Católica del Ecuador - Sede Ibarra (Ecuador). Correo electrónico: amculqui1@pucesi.edu.ec.
} 


\section{Introducción}

La Federación de Centros Awá del Ecuador (FCAE) ${ }^{1}$ fue beneficiada con una de las 14 frecuencias radiales comunitarias otorgadas por el gobierno central del Ecuador en el año 2009. La radio comunitaria "Ampara Su", denominada de esa manera una vez que se otorgó la frecuencia 90.7 FM, tiene un alcance para las provincias de Imbabura, zona sur del Carchi y norte de Pichincha.

El debate en torno a la necesidad de democratizar la palabra a través de la concesión de medios de comunicación a pueblos y nacionalidades del Ecuador, surgió con la aprobación de la Constitución del Ecuador en 2008. En uno de sus artículos transitorios, se exigía el desarrollo y aprobación de una ley que regulara la comunicación en el país. El 14 de junio de 2013 se aprobó en una histórica reunión la "Ley Orgánica de Comunicación" en el Ecuador después de más de dos años de debate en el pleno. Esta normativa poseía 119 artículos, 22 disposiciones transitorias, 6 reformatorias y 2 derogatorias $y$ en el Art. 106 se establece un reparto equitativo del espectro radioeléctrico: $33 \%$, medios públicos; $33 \%$ medios privados y $34 \%$, medios comunitarios. Con este antecedente se planteó un proyecto nacional que otorgaba radios comunitarias a las catorce nacionalidades del país, entre éstas la nacionalidad Awá del Ecuador. A partir de ello fue necesario seguir un debido proceso de investigación, organización y estructuración del medio que de forma inédita iba a otorgar micrófonos que permitirían amplificar voces y visibilizar la cultura del pueblo Awá

La presente investigación planteó, sobre un minucioso estudio, el camino a seguir en cuanto a lo estructuralorganizacional y estructural-informativo, por parte de la radio Ampara Su, ya que esta no poseía una estructura administrativa que coordinara roles, funciones $y$ dirigencia de la radio. También se construyó un plan de comunicación interno, externo, además un plan de auto sostenibilidad para que la radio fuese sostenible, una vez logrados todos los objetivos.

En lo que respecta a la estructura informativa, se construyó una parrilla de programación, acorde a las necesidades informativas de la población, algo que se logró a través de un diagnóstico y trabajo de campo, con lo cual pudimos sumergirnos en su cultura, cosmovisión y formas de vida, a fin de obtener una programación que visibilice su plan de vida y ayude a una mejor comunicación entre los pobladores de la nacionalidad.

${ }^{1}$ A partir de aquí, solo se llamará a la Federación por sus siglas.
En la zona norte del país en el año 2013, el 95\% de las radios eran radios comerciales, y el 5\% radios alternativas que no lograban mayor audiencia. (Vigil, 2012). Fue necesario contar con una radio comunitaria en la zona, en especial para la nacionalidad Awá, un sector vulnerable, que no poseía un canal para manifestarse, con el cual identificarse y que posibilitara crear las condiciones para un desarrollo local y sostenible. Con el planteamiento de este "modelo de gestión" fue posible que la Radio Comunitaria Ampara Su lograra un caminar a su propio ritmo, pero con una estructura más organizada, para que su accionar cumpla con los objetivos de un medio comunitario, pero sobre todo, que se desempeñe como la herramienta de comunicación que a la población Awá tanto le había hecho falta por años.

Los 22 centros Awá del Ecuador se encuentran ubicados en las tres provincias de la zona norte del país: 13 centros $^{2}$ en Carchi (Baboso, Gualpi Alto, Gualpi Bajo, Gualpi Medio, Guare, Ishpi, La Guaña, Ojala, Pailón, Río Tigre, Sabalera, San Marcos, Tarabita); 6 en Esmeraldas (Balsareño, Guadualito, La Unión, Mataje, Pambilar, Río Bogotá) y 3 en Imbabura (Palmira, Río Verde Bajo, Río Verde Medio).

Figura 1. Mapa del territorio Awá del Ecuador.

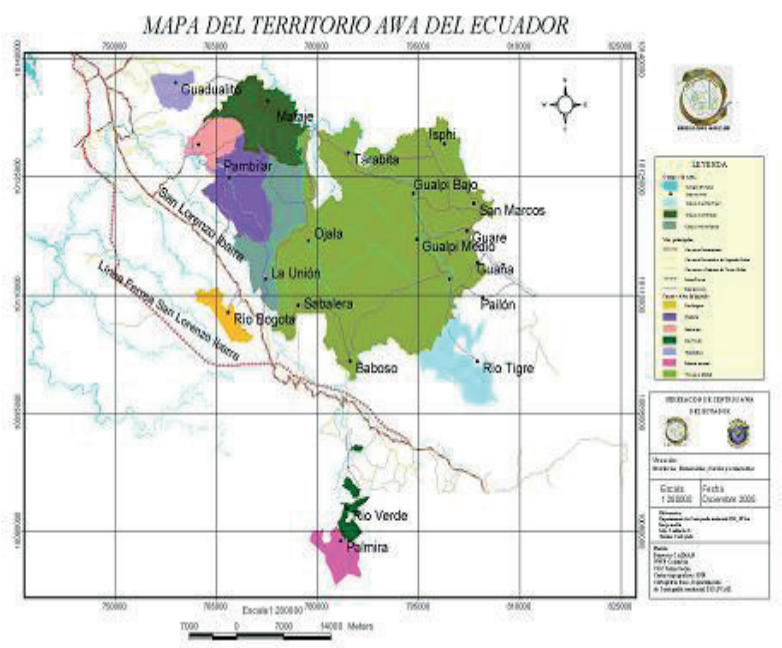

Fuente: http://federacionawa.org/elpuebloawa.htm

Los Awá son gente de montaña, su idioma es el Awapit, sus principales actividades económicas son la pesca, caza, agricultura, ganadería en pequeña escala y la elaboración de artesanías (FCAE, 2010). El pueblo Awá concibe al mundo dividido en cuatro espacios: el mundo del creador, mundo de los vivos, mundo de los muertos y mundo uchiawá o de los seres pequeños.

Para el pueblo Awá todo tiene vida: los ríos, las piedras,

2 El nombre de centros se denominó por el Ministerio de Bienestar Social, actual Ministerio de Inclusión Económica y social, al no cumplir los requisitos de ley para ser comunidad. 
el sol, la luna, entre otros. Conciben a las enfermedades relacionadas con los espíritus. Poseen rituales propios de sanación como el chutún, ojeado de la piedra, duende, espanto, etc. (FCAE, 2010). Están organizados a través de la Federación de Centros Awá del Ecuador, reconocida el 7 de septiembre del 2001 por el Ministerio de Bienestar Social en aquel año. El Consejo de la FCAE es elegido en un Congreso realizado cada tres años en la comunidad de San Marcos, se eligen además a los dirigentes de cada área de trabajo y de los centros territoriales. Permanentemente realizan asambleas territoriales con el propósito de analizar problemáticas internas y externas, con el propósito de evaluarlos y tratar de solucionarlos.

Con toda esta riqueza cultural y territorial que poseía la nacionalidad Awá, era fundamental un medio de comunicación que logre un canal de conexión entre comunidades a lo interno de su territorio y un canal de difusión de sus formas de vida al exterior, es por ello que el modelo de gestión para radios comunitarias, resultado de esta investigación, fue pionero en el Ecuador, ya que aportó para la presentación de requisitos formales para la asignación de la licencia definitiva por parte del Gobierno, e impulsando el proyecto de la Red de medios comunitarios, públicos, privados, locales que se originó a partir de la construcción de la Ley de Comunicación, que tipifica una redistribución de frecuencias para la democratización de la comunicación.

\section{Hacia una comunicación democrática}

En Latinoamérica, antes que la teoría, las experiencias en comunicación popular anticiparon la evolución de otros procesos que se advenían. Sin embargo, era cada vez más necesario un sustento teórico de acuerdo a lo que suscitaba en cada país. Las primeras experiencias de radios comunitarias en Colombia y Bolivia, marcaron el inicio de toda una concepción teórica denominada "Comunicación para el Desarrollo" a la que se fueron sumando varios autores desde diferentes enfoques y perspectivas para intentar describir los procesos que surgieron en América Latina desde la década del cincuenta del siglo XX.

La comunicación para el desarrollo, entiende que la comunicación y el desarrollo son dos esferas de la actividad humana íntimamente relacionadas; es decir, cualquier proyecto transformador conlleva un modo de entender la comunicación; $y$, asimismo, cada vez que nos comunicamos introducimos innovaciones en el entramado social.

De acuerdo a esto, la comunicación para el desarrollo es el vínculo histórico, teórico y procedimental entre los procesos comunicativos y la mejora de las condiciones de vida humana. Más específicamente, el campo se orienta a la planificación, ejecución y evaluación de estrategias de cambio social en el ámbito individual, social y medioambiental, ya sea con apoyo de una información de carácter instrumental o desde un enfoque eminentemente comunicativo.

De este concepto surge el planteamiento de que las comunidades deben ser actrices protagónicas de su propio desarrollo, de que la comunicación no debe ser necesariamente sinónimo de persuasión sino primordialmente mecanismo de diálogo horizontal e intercambio participativo y que, en vez de centrarse en forjar conductas individuales debe hacerlo en los comportamientos sociales conducentes, con los valores y las normas de las comunidades.

La comunicación tiene que partir no solamente de sus necesidades, sino también de las esperanzas de la gente, de sus sueños, propuestas y potencialidades. Para hablar de una comunicación social ligada al desarrollo, deben incorporarse esas utopías, porque tal vez esta sociedad no está cambiando dado que nos movemos en las esperanzas de otros y no las de la gente.

Un medio que trabaja por el desarrollo no puede quedarse en condenar a los políticos, a los parlamentarios, a los gobernantes. La política es necesaria, lo que pasa es que está mal manejada y, a través de los medios, debe darse a la política su verdadero sentido, ser un arte de la equidad en lugar de renunciar a ella y por ende al desarrollo y al cambio de la sociedad.

Luis Ramiro Beltrán, fue pionero en plantear desde la teoría a la Comunicación desde una concepción de "desarrollo", además cuestiona los modelos de difusión de innovaciones importados por la escuela funcionalista estadounidense. A través de sus investigaciones hizo un recuento de los modelos de comunicación tradicionales y jerárquicos importados desde Europa y occidente, pero que no tenían relación con los contextos latinoamericanos. La comunicación alternativa para el desarrollo democrático es la expansión y el equilibrio en el acceso de la gente al proceso de comunicación y en su participación en el mismo, empleando los medios - masivos, interpersonales y mixtos- para asegurar, además del avance tecnológico y del bienestar material, la justicia social, la libertad para todos y el gobierno de la mayoría (Beltrán, 2005:21).

La situación en América Latina a inicios de la década del 60 del siglo XX estuvo marcada por la Revolución Cubana (1959), que trajo consigo vientos libertarios en todo el continente y oportunidades de cambio con respecto a los oprimidos de países en supuesto subdesarrollo. Cabe 
mencionar que en esta época estaba presente también en el continente una fuerte corriente de pensamiento social con varios pensadores latinoamericanos, denominada como la Teoría de la dependencia, que denunciaban las brechas de desigualdad y dependencia económica de los países del sur con respecto al capital del norte (Prebisch 1963). Progreso y riqueza creciente para los menos y pobreza para los más (Gutiérrez 1973, p.131).

La “Teoría de la Dependencia” se llamó a este proceso que Beltrán describe en vender materia prima barata a los países desarrollados, para después comprar los productos manufacturados con costos más altos, produciendo un déficit crónico y creciente para los latinoamericanos, que derivó en procesos de subdesarrollo que acentuaban la desigualdad social. A partir de estas teorías, surgen reflexiones en distintos y heterogéneos ámbitos sociales. Por ejemplo, empiezan a surgir procesos alternativos de pensar a la comunicación desde las propias experiencias con los medios populares y alternativos de los países latinos.

En este contexto, surgen en América del Sur experiencias de comunicación popular, que intentan incluir en sus acciones a la población que ha sido objeto de discriminación y exclusión. Las radios de los mineros en Bolivia y la Radio Sutatenza en Colombia, fueron experiencias de medios populares que lograron cambiar el sentido de los medios tradicionales de comunicación, a pesar de sus diferencias en la gestión comunitaria y las formas de auto sostenimiento de las últimas. La radio había tomado en cuenta al pueblo, la gente lograba expresarse y escucharse a sí misma en un ejercicio de reconocimiento.

Radio Sutatenza y la iniciativa Acción Cultural Popular (ACPO) nacieron en 1947, como un proyecto de escuelas radiofónicas del sacerdote José Joaquín Salcedo y se convirtieron en una de las industrias culturales de mayor impacto social en América Latina hasta el cierre de la radio en 1989, sin embargo, ACPO continúa hasta la actualidad". Las "Radios Rebeldes" en Cuba o el movimiento del "Nuevo Cine Latinoamericano" en Bolivia denunciaron las condiciones de dominación y consiguen una emancipación de las estructuras de poder imperantes (Sel, 2009).

Es entonces que varios intelectuales inician con la Escuela Latinoamericana de Comunicación, a fin de realizar investigaciones y estudiar a la comunicación como un proceso que posibilita el cambio social, alejado de imposiciones y modelos que fracasaron en este proceso.

${ }^{3}$ Radio Sutatenza, la "escuela" de campesinos colombianos" El Espectador, 18 de abril de 2015, http://www.elespectador.com/noticias/ nacional/radio-sutatenza-escuela-de-campesinos-colombianosarticulo-555878
Se transforma en una diáspora intelectual, durante los años 70, reuniendo a los analistas del impacto social de los medios de comunicación de masa en los países de la región. Su contingente es formado por los pensadores que enfrentaron situaciones de exilio político, como el argentino Héctor Schmucler, el chileno Fernando Reyes Matta, el uruguayo Roque Faraone y el peruano Rafael Roncagliolo; por figuras notables invitadas por las organizaciones internacionales, como el boliviano Luis Ramiro Beltrán, el venezolano Antonio Pasquali, el paraguayo Juan Díaz Bordenave; o por inmigrantes intelectuales del calibre del belga Armand Mattelart, del español Jesús Martín Barbero o de la estadounidense Elizabeth-Fox. (Marques, 2007)

Desde esta perspectiva de Comunicación para eldesarrollo, comienza la teorización también de la comunicación popular y los medios comunitarios, aterrizando muchos años después de la unión de luchas sociales e intelectuales colectivos en políticas públicas de comunicación y marcos regulatorios de la comunicación, que se implementaron en los países de América Latina, en los que intervinieron gobiernos progresistas y de izquierda.

Varios teóricos contribuyeron al replanteamiento del concepto de comunicación, pero muy pocos se dedicaron de lleno a este oficio, brindando pocos modelos de comunicación democrática que compitan con los tradicionales jerárquicos ya establecidos. Con base en las teorías de Freire; Cloutier (1973) planteó el esquema "Emirec" que conjugaba al emisor y al receptor. Gerace (1973), exploró aún más la naturaleza de la "comunicación horizontal” y Gutiérrez (1973), que escribió sobre la noción del lenguaje total.

\section{Modelo de Comunicación Horizontal}

La Escuela Latinoamericana de Comunicación objetabalos esquemas verticales tradicionales, su principal exponente fue Luis Ramiro Beltrán, quien planteó el "Modelo de Comunicación Horizontal", un modelo distinto a los modelos funcionalistas que se venían estudiando en las aulas universitarias y donde el principal protagonista era el "diálogo", como catalizador de los procesos y de las relaciones sociales democráticas y participativas.

Varios autores de la Escuela Latinoamericana buscaban fundamentos para percibir a la sociedad como movimiento. Este modo de concebir a la sociedad contribuye al 
fortalecimiento y consolidación de la propuesta de comunicación horizontal, que se dio a conocer también como comunicación popular, participativa y/o alternativa (Peruzzo, 2000).

Figura 2. Modelo de Comunicación Horizontal.

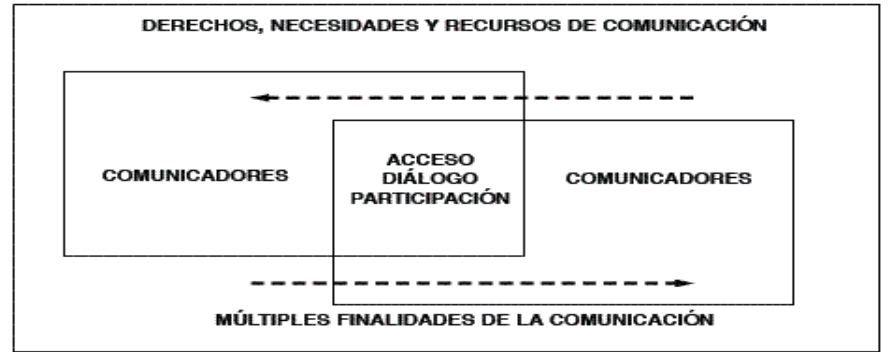

Fuente: Beltrán (2007).

\section{Metodología}

El presente estudio presenta una alineación metodológica cuantitava-cualitativa, por cuanto se desarrollan los siguientes tipos de investigación:

a. Inductivo-deductivo: Con el uso de este método, se partió del hecho puntual de que la radio Ampara $\mathrm{Su}$ carecía de una estructura. A partir de ello se establecieron generalidades que fundamentaron la investigación. Se analizaron experiencias particulares de varias organizaciones que se dedican a la radiodifusión comunitaria y el diagnóstico de campo con la población Awá, a fin de concluir en la problemática: falta de un modelo de gestión para las radios comunitarias en el Ecuador.

b. Analítico-cualitativo: Este método permite establecer las premisas fundamentales y necesarias para la Radio Ampara $\mathrm{Su}$ en su aspecto estructural e informativo. Se analizó la información que fue útil como datos, contenidos, bibliografía e investigación de campo de forma cualitativa para determinar las características etnográficas de la población Awá.

Las técnicas utilizadas fueron:

a. Encuesta: Se diseñó un modelo de encuesta que fue aplicado a la muestra calculada para determinar el contexto de la población de la nacionalidad Awá frente a la radio, y conocer sus costumbres, cultura, cosmovisión y lograr construir el modelo de gestión. Los datos resultantes de la encuesta fueron tabulados, graficados y debidamente interpretados.

b. Entrevista: Para ampliar la información y conocer experiencias de instituciones que trabajan y gestionan radios comunitarias se realizaron entrevistas a los representantes del Centro Internacional de Estudios Superiores de Comunicación para América Latina (CIESPAL), Coordinadora de Radio Popular Educativa del Ecuador (CORAPE), Escuelas Radiofónicas Populares del Ecuador (ERPE), Asociación Latinoamericana de Educación Radiofónica (ALER) para poder aprender de sus gestiones y adaptarlas a la Radio Ampara Su.

c. Ficha de Observación: Con el propósito de conocer más sobre el contexto de la población Awá realizamos fichas de observación para establecer información cualitativa como la participación de la gente en las asambleas, el número de mujeres asistentes. Además de información pertinente a las comunidades como si poseen o no escuela, Sub Centro de salud, vías de acceso, etc.

\section{Población y universo}

La población aproximada de la nacionalidad Awá presente en las tres provincias del Ecuador es de 4.795 habitantes (FCAE, 2010) distribuidas de la siguiente manera:

- Provincia del Carchi, Cantón Tulcán, Parroquia Tobar Donoso y Maldonado: 2.000 habitantes.

- Provincia de Imbabura, en las riberas de los ríos: Verde, Lita, Buenos Aires: 795 habitantes.

- Provincia de Esmeraldas, ubicadas en las zonas de Palabí, Mataje, Pulubí: 1.000 habitantes pertenecientes al Cantón San Lorenzo.

\section{Muestra}

Se aplicaron 348 encuestas en cinco visitas a las comunidades de Río Verde Bajo, El Baboso, Pambilar, El Pailón y San Marcos, pertenecientes a las tres provincias que nos permitieron conocer el contexto cultural de la población Awá, así como sus gustos musicales y el tipo de programación que deseaban escuchar en su radio.

\section{Matriz de Relación}

Se elaboró una matriz de relación partiendo desde los objetivos diagnósticos, para organizar la información que se deseaba obtener de acuerdo a las variables, indicadores y técnicas de recolección de datos. Después se aplicaron las encuestas y con la información diagnóstica obtenida, se trabajó junto a estas mismas comunidades una matriz de análisis FODA para determinar estrategias de trabajo entre la población y la radio. 
Tabla 1. Relación de objetivos, variables, indicadores, técnicas, fuentes

\begin{tabular}{|c|c|c|c|c|}
\hline $\begin{array}{l}\text { Objetivos } \\
\text { diagnósticos }\end{array}$ & Variables & Indicadores & Técnicas & $\begin{array}{l}\text { Fuente de } \\
\text { información }\end{array}$ \\
\hline $\begin{array}{l}1 . \\
\text { Conocer la } \\
\text { situación social } \\
\text { y cultural de la } \\
\text { población Awá. }\end{array}$ & $\begin{array}{c}\text { Situación So- } \\
\text { cial y Cultural } \\
\text { de la población } \\
\text { Awá. }\end{array}$ & $\begin{array}{l}\text {-Situación laboral } \\
\text {-Nivel de } \\
\text { escolaridad } \\
\text {-Problemas } \\
\text { familiares } \\
\text {-Factores sociales } \\
\text { - Factores culturales } \\
\text { y tradicionales } \\
\text {-Forma de } \\
\text { organización }\end{array}$ & $\begin{array}{c}\text { Encuesta } \\
\text { Observación }\end{array}$ & $\begin{array}{l}\text { - Población de la na- } \\
\text { cionalidad Awá. } \\
\text { - Dirigentes de las } \\
\text { comunidades. } \\
\text {-Presidente de la } \\
\text { FCAE }\end{array}$ \\
\hline $\begin{array}{l}2 . \\
\text { Identificar la } \\
\text { importancia } \\
\text { de una radio } \\
\text { comunitaria en la } \\
\text { sociedad. }\end{array}$ & $\begin{array}{l}\text { Importancia } \\
\text { de la radio } \\
\text { comunitaria. }\end{array}$ & $\begin{array}{l}\text {-Beneficios de } \\
\text { un medio de } \\
\text { comunicación } \\
\text { alternativo. } \\
\text {-La necesidad } \\
\text { de contar con } \\
\text { una radio en las } \\
\text { comunidades. } \\
\text {-Expectativa de } \\
\text { las comunidades } \\
\text { frente a una radio } \\
\text { comunitaria. } \\
\text {-Uso de una radio } \\
\text { comunitaria. }\end{array}$ & $\begin{array}{c}\text { Asambleas } \\
\text { Entrevista } \\
\text { Documental }\end{array}$ & $\begin{array}{l}\text {-Población Awá } \\
\text {-Dirigentes } \\
\text {-Presidente la FCAE } \\
\text { - Especialistas en Ra- } \\
\text { dio Comunitaria }\end{array}$ \\
\hline $\begin{array}{l}3 . \\
\text { Analizar } \\
\text { el proceso } \\
\text { administrativo y } \\
\text { estructural en la } \\
\text { radio Ampara-Su. }\end{array}$ & $\begin{array}{c}\text { Proceso } \\
\text { administrativo } \\
\text { y estructural } \\
\text { de la radio } \\
\text { Ampara-Su. }\end{array}$ & $\begin{array}{l}\text {-Organigrama } \\
\text {-Manual de } \\
\text { funciones } \\
\text {-Convenios } \\
\text { - Toma de } \\
\text { decisiones } \\
\text {-Pautaje }\end{array}$ & $\begin{array}{l}\text { Entrevista } \\
\text { Observación } \\
\text { de Campo }\end{array}$ & $\begin{array}{l}\text { - Población de la na- } \\
\text { cionalidad Awá. } \\
\text { - Dirigente de la } \\
\text { radio } \\
\text {-Presidente de la } \\
\text { FCAE }\end{array}$ \\
\hline $\begin{array}{l}4 . \\
\text { Determinar } \\
\text { el grado de } \\
\text { incidencia de la } \\
\text { programación } \\
\text { radial de } \\
\text { la emisora } \\
\text { Ampara-Su, en } \\
\text { las comunidades } \\
\text { Awá. }\end{array}$ & $\begin{array}{l}\text { Programación } \\
\text { Radial }\end{array}$ & $\begin{array}{l}\text {-Preferencias } \\
\text { musicales } \\
\text {-Secciones } \\
\text {-Calidad de la } \\
\text { Información } \\
\text { - Nivel de educación } \\
\text { - Manejo de fuentes } \\
\text { de información } \\
\text { - Temáticas } \\
\text {-Grado de } \\
\text { Participación }\end{array}$ & Encuesta & $\begin{array}{l}\text { - Población de la na- } \\
\text { cionalidad Awá. } \\
\text { - Dirigentes de las } \\
\text { comunidades } \\
\text {-Presidente de la } \\
\text { FCAE }\end{array}$ \\
\hline $\begin{array}{l}5 . \\
\text { Identificar la } \\
\text { cobertura con } \\
\text { la que cuenta la } \\
\text { radio Ampara-Su } \\
90.7\end{array}$ & $\begin{array}{l}\text { Cobertura } \\
\text { de la radio } \\
\text { Ampara-Su }\end{array}$ & $\begin{array}{l}\text { - Mapeo en las } \\
\text { comunidades que } \\
\text { receptan la señal } \\
\text { de la radio } 90.7 \\
\text { Ampara-Su. } \\
\text {-El Mapa ideal de } \\
\text { cobertura } \\
\text {-Antenas } \\
\text {-Equipos }\end{array}$ & Observación & $\begin{array}{l}\text { - Dirigente de la } \\
\text { radio } \\
\text {-Presidente la FCAE } \\
\text { - Dirigente de la } \\
\text { Radio } \\
\text { - Trabajadores de la } \\
\text { radio }\end{array}$ \\
\hline
\end{tabular}

Fuente: elaboración propia.

\section{Resultados y Discusión}

La encuesta contó con diez preguntas, el método de aplicación varió de acuerdo al territorio, en varias comunidades se intervino en sus asambleas generales a manera de taller, en otras comunidades se lo hizo de manera individual y varias veces con la participación de un intérprete que hablara awapit; dado que la encuesta estaba en español fue necesario traducirla para que la población comprenda y responda en su propio idioma.
Tabla 2. Matriz resumen de encuesta aplicada

\begin{tabular}{|c|c|c|c|}
\hline Pregunta & Opción & Frecuencia & Porcentaje \\
\hline $\begin{array}{c}1 . e^{\text {Tiene usted }} \\
\text { radio en su casa? }\end{array}$ & $\begin{array}{l}\text { Sí } \\
\text { No }\end{array}$ & $\begin{array}{l}317 \\
31\end{array}$ & $\begin{array}{l}91 \% \\
9 \%\end{array}$ \\
\hline $\begin{array}{c}\text { 2. ¿Cuánto tiempo } \\
\text { en el día escucha } \\
\text { radio? }\end{array}$ & $\begin{array}{l}\text { Menos de una hora } \\
\text { 1-3 horas } \\
5 \text { o más horas }\end{array}$ & $\begin{array}{l}49 \\
156 \\
143\end{array}$ & $\begin{array}{l}14 \% \\
45 \% \\
41 \%\end{array}$ \\
\hline $\begin{array}{c}\text { 3. ¿En qué horarios } \\
\text { escucha usted la } \\
\text { radio? }\end{array}$ & $\begin{array}{l}\text { En la mañana } \\
\text { (Desde } 5 \mathrm{am} \text { hasta } \\
\text { 11am) } \\
\text { En la tarde (Desde } \\
12 \mathrm{pm} \text { hasta } 5 \mathrm{pm}) \\
\text { En la noche (Desde } \\
6 \mathrm{pm} \text { hasta } 10 \mathrm{pm})\end{array}$ & $\begin{array}{l}149 \\
\\
152 \\
192\end{array}$ & $\begin{array}{r}30 \% \\
\\
\\
31 \% \\
39 \%\end{array}$ \\
\hline $\begin{array}{l}\text { 4. Las radios } \\
\text { que llegan a su } \\
\text { comunidad son en } \\
\text { su mayoría: }\end{array}$ & $\begin{array}{l}\mathrm{AM} \\
\mathrm{FM}\end{array}$ & $\begin{array}{l}35 \\
313\end{array}$ & $\begin{array}{l}10 \% \\
90 \%\end{array}$ \\
\hline $\begin{array}{c}\text { 5. ¿Qué radios } \\
\text { que llegan a su } \\
\text { comunidad escucha } \\
\text { Usted? }\end{array}$ & $\begin{array}{l}\text { Negra Latina } \\
\text { Zaracay } \\
\text { HCJB } \\
\text { Galaxia } \\
\text { Voz de los Andes } \\
\text { Camawari } \\
\text { Colombia Estéreo } \\
\text { Voz } 2 \text { Amigos } \\
\text { Estéreo Oriente } \\
\text { Otras Radios }\end{array}$ & $\begin{array}{l}225 \\
66 \\
62 \\
62 \\
85 \\
72 \\
90 \\
62 \\
77 \\
83\end{array}$ & $\begin{array}{l}25 \% \\
8 \% \\
7 \% \\
7 \% \\
10 \% \\
8 \% \\
10 \% \\
9 \% \\
9 \%\end{array}$ \\
\hline $\begin{array}{l}\text { 6. ¿Qué tipo de } \\
\text { programas le } \\
\text { gustaría escuchar en } \\
\text { radio Ampara Su? }\end{array}$ & $\begin{array}{l}\text { Noticia } \\
\text { Entrevistas } \\
\text { A su Gente } \\
\text { Deportes } \\
\text { Agricultura } \\
\text { Salud } \\
\text { Seguridad } \\
\text { Educación } \\
\text { Entretenimiento } \\
\text { Otros programas }\end{array}$ & $\begin{array}{l}300 \\
236 \\
272 \\
178 \\
183 \\
204 \\
251 \\
257 \\
168 \\
46\end{array}$ & $\begin{array}{l}14 \% \\
11 \% \\
13 \% \\
9 \% \\
9 \% \\
10 \% \\
12 \% \\
12 \% \\
8 \% \\
2 \%\end{array}$ \\
\hline $\begin{array}{l}\text { 7. ¿Qué idioma le } \\
\text { gustaría escuchar en } \\
\text { la radio Ampara Su? }\end{array}$ & $\begin{array}{l}\text { Español } \\
\text { Awapit } \\
\text { Ambos }\end{array}$ & $\begin{array}{l}26 \\
55 \\
267\end{array}$ & $\begin{array}{l}7 \% \\
16 \% \\
77 \%\end{array}$ \\
\hline
\end{tabular}




\begin{tabular}{|c|c|c|c|}
\hline $\begin{array}{l}\text { 8. Considera que la } \\
\text { implementación de } \\
\text { la radio comunitaria } \\
\text { Ampara Su para la } \\
\text { nacionalidad Awá } \\
\text { es: }\end{array}$ & $\begin{array}{l}\text { Poco Importante } \\
\text { Importante } \\
\text { Muy Importante }\end{array}$ & $\begin{array}{l}12 \\
72 \\
264\end{array}$ & \begin{tabular}{|l|}
$3 \%$ \\
$21 \%$ \\
$76 \%$
\end{tabular} \\
\hline $\begin{array}{l}\text { 9. ¿Le gustaría } \\
\text { participar en la } \\
\text { radio? }\end{array}$ & $\begin{array}{l}\text { SI } \\
\text { NO }\end{array}$ & $\begin{array}{l}265 \\
83\end{array}$ & $\begin{array}{l}76 \% \\
24 \%\end{array}$ \\
\hline $\begin{array}{c}\text { 10. ¿Qué tipo de } \\
\text { música le gustaría } \\
\text { escuchar en la radio } \\
\text { Ampara Su? }\end{array}$ & $\begin{array}{l}\text { Cumbia } \\
\text { Marimba } \\
\text { Bombas } \\
\text { Guitarra } \\
\text { San Juanitos } \\
\text { Pasillo } \\
\text { Bachata } \\
\text { Vallenato } \\
\text { Música cristiana } \\
\text { Baladas } \\
\text { Música local } \\
\text { Otro }\end{array}$ & $\begin{array}{l}289 \\
269 \\
207 \\
121 \\
118 \\
130 \\
88 \\
115 \\
74 \\
122 \\
229 \\
40\end{array}$ & \begin{tabular}{|l|}
$16 \%$ \\
$15 \%$ \\
$11 \%$ \\
$7 \%$ \\
$7 \%$ \\
$7 \%$ \\
$5 \%$ \\
$6 \%$ \\
$4 \%$ \\
$7 \%$ \\
$13 \%$ \\
$2 \%$
\end{tabular} \\
\hline
\end{tabular}

Fuente: elaborado por: las autoras.

\section{FODA}

\section{FORTALEZAS:}

a. La radio Ampara-Su tiene un buen nivel de aceptación en las comunidades Awá, pues es el primer medio de comunicación que posee la nacionalidad.

b. La radio es un ente de participación y socialización de la cultura.

c. En el aspecto económico la radio brinda costos de pautaje accesibles.

d. La radio cuenta con tecnología de punta.

\section{OPORTUNIDADES:}

a. Asignación de frecuencias comunitarias por parte del Gobierno Nacional.

b. La radio cuenta con el apoyo económico de la Secretaría de la Gestión de la Política, y apoyo técnico de otras instituciones.

c. Poder visibilizar su idioma como fortaleza y cultura propia

d. Varias ONGs e instituciones que coinciden con la ideología de la radio quieren tener un espacio en la radio y pautar.

\section{DEBILIDADES:}

a. No se cuenta con la cobertura completa para todas las 22 comunidades.

b. Falta de conocimiento de la población Awá en producción y gestión de medios de comunicación.

\section{AMENAZAS:}

a. Hay otras emisoras que llegan a las comunidades Awá.

b. Existe desconocimiento por parte de las personas que no son Awá, acerca de la cultura de su nacionalidad.

\section{ESTRATEGIAS FA, FO, DO, DA}

\section{ESTRATEGIAS FA}

a. $\mathbf{F}(\mathrm{a}) \mathbf{A}(\mathrm{a})$ : Aprovechar el interés y aceptación que tiene la población Awá, frente a su radio comunitaria para desarrollar programación interesante, el $50 \%$ en awapit y el $50 \%$ en español, con la finalidad de conseguir la identificación de los pobladores Awá frente a este medio de comunicación.

b. F(a) A (b): Desarrollar programas para visibilizar la cultura Awá.

c. $\mathbf{F}(\mathrm{b}) \mathbf{A}(\mathrm{b})$ : Potenciar la participación tanto de la población Awá y de todos los ciudadanos.

d. $\quad \mathbf{F}(\mathrm{c}) \mathbf{A}(\mathrm{b})$ : Implementar espacios atractivos para ofertar el respectivo pautaje.

\section{ESTRATEGIAS FO}

a. $\quad \mathbf{F}(\mathrm{a}) \mathbf{O}(\mathrm{a})$ : Gestionar la implementación de otra antena repetidora, a fin de contar con cobertura para las 22 comunidades, a través del Gobierno ecuatoriano.

b. $\mathbf{F}(\mathrm{b}) \mathbf{O}(\mathrm{c})$ : Generar interés en la programación radial en toda la ciudadanía, a través de la utilización del idioma awapit.

c. $\quad \mathbf{F}(\mathrm{c}) \mathbf{O}(\mathrm{d})$ : Promocionar el pautaje con otras instituciones que coincidan con la ideología de la radio comunitaria. 


\section{ESTRATEGIAS DO}

- D(a) O (b): Diseñar el estudio para la implementación de otra antena, a través de la Secretaría de la Gestión de la Política.

- D (b) O(a): Capacitar a población Awá en producción y gestión de medios de comunicación, mediante la gestión con el Gobierno para conseguir becas y financiamiento para recibir talleres.

\section{ESTRATEGIAS DA}

- D(a) A(a): Reiterar la necesidad de tener una adio comunitaria propia para la nacionalidad Awá, realizando programación acorde al interés de este pueblo.

- D(a) A (b): Proponer una parrilla de programación que permita interactuar tanto con las personas de nacionalidad Awá y la ciudadanía que recepta la señal de la radio Ampara-Su.

\section{Discusión de Resultados}

Luego de haber aplicado las respectivas encuestas y analizado las fortalezas, oportunidades, debilidades y amenazas (FODA) se pudo determinar la necesidad de implementar verdaderos procesos comunicacionales, administrativos, estructurales, económicos, que permitan consolidar a la emisora Ampara-Su, como un medio alternativo y comunitario, para gestar la participación y visibilizar al pueblo Awá, que por años ha sido relegado.

Un 91\% de población encuestada tiene una radio en su casa y la escucha de 3 a 5 horas diarias, las personas manifiestan que el horario en el que más escuchan radio es en la noche y lo que más les gustaría escuchar son noticias de sus comunidades y fuera de ellas, tanto en español como en awapit, prevaleciendo su lengua para visibilizarla. La gente, en su mayoría, piensa que la implementación de la radio en sus comunidades es "muy importante", y además están dispuestos a participar en la programación de la radio.

Los resultados fueron alentadores, pues la gente estaba motivada a impulsar su medio de comunicación, por ello fue importante poner en marcha un Modelo de Gestión, resultado de la presente investigación, dado que por más de tres años la radio estuvo al aire sin producción, programación y organización interna.

Es preciso que la comunidad Awá, tenga su propio medio para la difusión de su cultura y la implementación de procesos democráticos y participativos, con la finalidad de permitir el desarrollo social, económico de esta nacionalidad; fomentando el respeto a su cultura y diversidad.

\section{Conclusiones}

La estructuración de la Radio Comunitaria Ampara Su 90.7 cumplió primordialmente el objetivo de visibilizar a la Nacionalidad Awá dentro del contexto nacional, por lo que consideramos un impacto positivo, pues por muchos años, su historia ha sido invisibilizada y no tomada en cuenta por autoridades, instituciones y la población en general que no conocía de la existencia de esta nacionalidad y su territorio.

La población Awá no contaba antes con un medio de comunicación que permita interrelacionarse entre comunidades y que acorte distancias entre sus territorios, por lo que fue importante que al ser la radio la única tecnología y canal por donde se informan de noticias, avisos, comunicados entre comunidades (debido a la lejanía) este trabajo fue una excelente oportunidad para reafirmar su cultura, fortalecer su organización y difundir sus ideas.

El awapit como lengua propia de la nacionalidad Awá, fue visibilizado a través de la radio, la población se reconocía en sus propias voces y la construcción de identidad cultural hizo que sus creencias, costumbres y tradiciones no se pierdan.

A través del Modelo de Gestión, estructurado a raíz de esta investigación, la programación de la Radio Ampara $\mathrm{Su}$, fue producida desde su propio contexto y necesidades, donde la audiencia se convirtió en protagonista de sus contenidos, planteando un real ejercicio de comunicación horizontal, manteniendo la interacción activa entre los locutores y el equipo de la radio, cambiando el papel de meros receptores a partícipes activos que emitían una retroalimentación y demandas informativas.

La Nacionalidad Awá del Ecuador, relegada del contexto nacional, ha sido muchas veces abandonada en temas críticos queles afecta a diario, comolasminerasy madereras, la falta de servicios básicos, legalización de tierras, alto índice de desnutrición en los niños y analfabetismo en la población en general; han sido problemas que a pesar que cuentan con la FCAE, organización que los acompaña, no siempre lograron cumplir con la demanda de todas estas necesidades. Es por ello que consideramos que el resultado de la presente investigación generó un gran impacto en la población, pues al contar con un medio de comunicación propio se puede insistir a las autoridades e instituciones pertinentes para que no dejen de lado todas 
estas demandas y luchas de la nacionalidad.

\section{Referencias}

1. Beltrán, L. R. (2005). La Comunicación para el Desarrollo en Latinoamérica: Un recuento de medio siglo. III Congreso Panamericano de la Comunicación (pág.15). Buenos Aires: s/e.

2. Beltrán, L. R. (2007). Adiós Aristóteles: La Comunicación Horizontal. (A. L. Comunicación., Ed.) Revista Latinoamericana de Ciencias de la Comunicación, 15- 35.

3. Cloutier, J. (1973). La Communication AudioScripto-Visuelle. Montreal: Presses Universitaires.

4. FCAE. (2010). Plan de Vida. Ecuador: Federación de Centros Awá del Ecuador.

5. Federación de centros Awá del Ecuador. (2002). La historia del Pueblo Awá. Quito: Efecto Gráfico.

6. Gerace, F. (1973). Comunicación Horizontal. Lima: Librería Studium.

7. Gutiérrez, F. (1973). El Lenguaje Total: Una Pedagogía de los Medios de Comunicación. Buenos Aires: Editorial Humanitas.

8. Marqués, J. (2007). Reto de la investigación latinoamericana en comunicación. Revista Latinoamericana de Comunicación Chasqui. Quito: CIESPAL.
9. Peruzzo, C. M. (2000). Escuelas Latinoamericana de Comunicación: contribuciones de Luis Ramiro Beltrán. En V. autores, Investigación sobre Comunicación en Latinoamérica. Inicio, trascendencia y proyección. (pág. 164). Bolivia: Plural z Editores.

10. Prebisch, R. (1963). Hacia una dinámica del desarrollo latinoamericano. Con un apéndice sobre el falso dilema entre desarrollo económico y estabilidad monetaria. México: Fondo de Cultura Económica

11. Sel, S. (2009). Comunicación alternativa y políticas públicas en el combate latinoamericano en La Comunicación mediatizada: hegemonías, alternatividades, soberanías. Recuperado de $\quad$ http://biblioteca.clacso.edu.ar/clacso/ gt/20160229044939/02sel.pdf Buenos Aires: CLACSO.

12. Vigil, J. I. (08 de Noviembre de 2012). Radios Comunitarias: Una historia de discriminaciones. (CIESPAL, Entrevistador) 